\title{
Probing Dielectric Properties of Metal-Organic Frameworks: MIL-53(Al) as a Model System for Theoretical Predictions and Experimental Measurements via Synchrotron Far- and Mid- InfraRed Spectroscopy
}

\author{
Kirill Titov, ${ }^{a}$ Zhixin Zeng, ${ }^{a}$ Matthew R. Ryder, ${ }^{a}$ Abhijeet K. Chaudhari, ${ }^{a}$ Bartolomeo Civalleri, ${ }^{b}$ \\ Chris S. Kelley,,$^{c}$ Mark D. Frogley, ${ }^{c}$ Gianfelice Cinque, ${ }^{c}$ and Jin-Chong Tan ${ }^{a}$ \\ ${ }^{a}$ Multifunctional Materials and Composites (MMC) Laboratory, Department of Engineering \\ Science, University of Oxford, Parks Road, Oxford, OX1 3PJ, UK \\ ${ }^{b}$ Department of Chemistry, NIS and INSTM Reference Centre, University of Turin, via Pietro \\ Giuria 7, 10125 Torino, Italy \\ ${ }^{c}$ Diamond Light Source, Harwell Campus, Chilton, Oxford, OX11 0DE (UK) \\ *E-mail: jin-chong.tan@eng.ox.ac.uk
}


Abstract: Emerging nanoporous materials, such as metal-organic frameworks (MOFs), are promising low- $k$ dielectrics central to next-generation electronics and high-speed communication. Hitherto, the dielectric characterization of MOFs is scarce, with very limited experimental data for guiding new materials design and synthesis. Herein we demonstrate the efficacy of high-

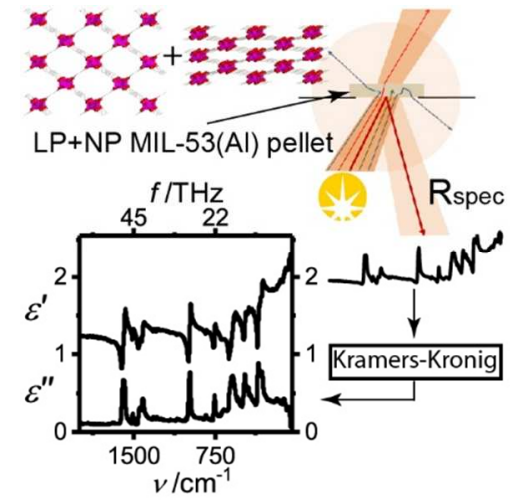
resolution synchrotron infrared (IR) specular reflectance experiments, to study the dynamic dielectric properties of a flexible MOF structure: bi-stable MIL-53(Al) that exhibits switching between a large pore (LP) and a narrow pore (NP) architecture. We show the ratio of LP:NP content of a polycrystalline sample can be changed via increased mechanical stress applied for pelletizing the MIL-53(Al) powder. We quantify the frequency-dependent dielectric constants over $\sim 1$ to $120 \mathrm{THz}$, identifying all dielectric transitions as a function of stress and phase mixtures, showing how porosity modifies MOF's dielectric properties.

Porous metal-organic frameworks (MOFs) are hybrid materials renowned for their large surface area, ${ }^{1-2}$ accompanied by remarkable structural flexibility ${ }^{3-4}$ and framework dynamics ${ }^{5-7}$ in response to diverse physical and chemical stimuli. ${ }^{8}$ Traditionally, the development of MOFs has been instigated by potential applications such as gas storage, $\mathrm{CO}_{2}$ sequestration and catalysis, destined for familiar microporous materials like zeolites. ${ }^{9-10}$ More recently, however, the research focus is shifting towards the exploration of MOFs to accomplish technological applications associated with electronics and photonics, ${ }^{11}$ optoelectronics, ${ }^{12-13}$ smart switches and sensors. ${ }^{14-16}$ On the one hand, there has been a rapidly growing body of work concerning electrically conducting MOFs; ${ }^{17-18}$ but on the other hand substantially less attention has been 
devoted to their dielectric properties, ${ }^{19}$ which are important for future telecommunications, microelectronics and photonics applications.

Theoretical calculations $^{20}$ and a limited set of static dielectric measurements reveal that MOFs are highly promising "low- $k$ " dielectric materials $(k \sim 2$ to 5$),{ }^{19}$ owing to their porosity and tunable chemical and structural versatilities. Next-generation microelectronics with an operating frequency exceeding $10^{9} \mathrm{~Hz}(\mathrm{GHz})$ and high-speed terahertz $(\mathrm{THz})$ communication technologies $\left(10^{12} \mathrm{~Hz}\right.$ and beyond) will require the implementation of new low- $k$ dielectric materials, replacing the classical $\mathrm{SiO}_{2}(k \sim 4)$ to minimize electronic cross-talk, signal delays and power losses. ${ }^{19,21}$ Only a few experiments have been reported to date on the dielectric behavior of MOF-based materials. For example, Eslava et $a .^{22}$ employed impedance spectroscopy with a capacitor arrangement to measure the dielectric constant of a micrometer-thick ZIF-8 polycrystalline film, and determined a relatively low $k$-value of $\sim 2.3$ across the frequencies of $100 \mathrm{~Hz}$ to $1 \mathrm{MHz}$. Likewise, Lu et al. ${ }^{23}$ applied the impedance method to measure the dielectric constants of a Sr-based MOF, where the dehydrated sample has $k \sim 2.4$ at under $10 \mathrm{kHz}$. In addition to the examples above obtained at frequencies below $1 \mathrm{MHz}$, Redel et al. ${ }^{24}$ used spectroscopic ellipsometry to study the variation in the refractive index (n) of HKUST-1 films in the visible wavelength, from which the dielectric constants have been estimated by $k=n^{2}$. Noteworthy, frequency-dependent dielectric functions $\tilde{\varepsilon}(\omega)$ of selected Zn-based MOFs have been computed using density functional theory (DFT) up to the near ultraviolet (UV) spectral range. ${ }^{25}$ Yet, there is no experimental studies about MOF dielectric characteristics in the higher frequency region of $\sim \mathrm{THz}$ (far- and mid-IR), fundamental to the development of emergent wireless communications, electronics and optical sensors. 
Herein we describe the novel application of a high-resolution synchrotron IR specular reflectance method ${ }^{26}$ (Beamline B22 MIRIAM, Diamond Light Source) to study the dynamic dielectric characteristics of the MIL-53(Al) polycrystalline powder, encompassing the broad spectral range of 1.2 to $120 \mathrm{THz}$. The structural bistability of MIL-53(Al) is well-documented, ${ }^{4}$ existing in two structural configurations viz. large pore (LP) and narrow pore (NP) architectures, as depicted in Fig.1a. Switching between the LP $\rightleftharpoons$ NP structures can be triggered by water/solvent uptake, temperature swing or mechanical stress (pressure). ${ }^{27-28}$ This provides us with the unique opportunity to monitor the variation in dielectric properties, in which the ratio of LP:NP phase mixtures can be tuned by controlling the externally applied stress.

The as-received MIL-53(Al) powder was used to make 9 pellets at increasing pressures, see Fig.1b. It is convenient to refer to the pellets in terms of the mass (in metric ton, $t$ ) applied to press each pellet, i.e. the $0.1 \mathrm{t}$ pellet or the $10 \mathrm{t}$ pellet, rather than the uniaxially applied nominal stress (force/area) $7.39 \mathrm{MPa}$ and $739 \mathrm{MPa}$, respectively. Fig. 1c shows the nominal density of the pellets as a function of the applied stress. The pellet density appears to follow a logarithmic law (with the $8 \mathrm{t}$ pellet being an outlier as a result of part of the pellet being chipped off), and approaching $\sim 90 \%$ of the theoretical single-crystal density of NP MIL-53(Al) at $\sim 800 \mathrm{MPa}$. This increase in density indicates a drastic decrease in void size and better packing of the crystals inside the pellets (see SI Fig. S6 for a schematic representation). Crystal size is an important consideration here and we note that the supplied MIL-53(Al) crystals vary in size from $50 \mathrm{~nm}$ up to $2 \mu \mathrm{m}$ (see SI Fig. S2 for SEM of the as-received powder). This distribution of crystal sizes is comparable to the wavelength of incident light within the spectral region of interest, thus some diffraction of the incident beam can be occurring; see SI Fig. S7 for a breakdown of what 
happens to various parts of the incident beam. Here we note that it is predominantly the specular reflected light that was measured.

a. Large Pore (LP) Narrow Pore (NP)

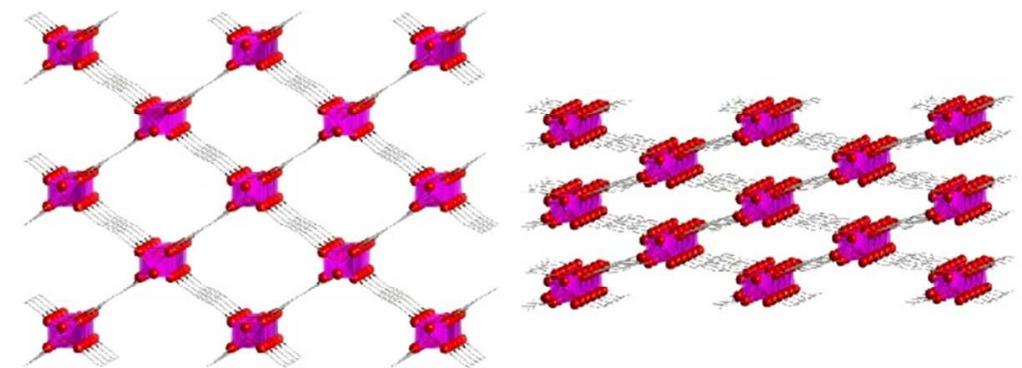

b.

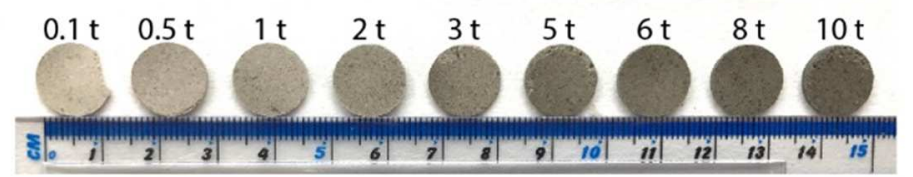

c.

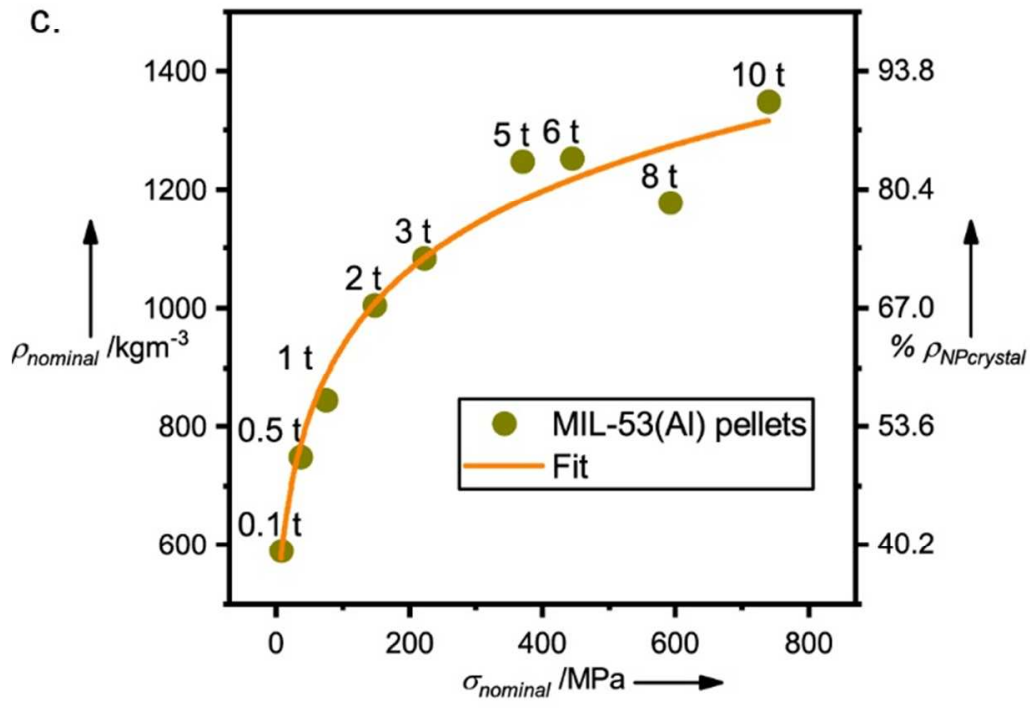

Figure 1. MIL-53(Al) pellet composition: (a) Schematic illustration of the crystal structure of MIL-53(Al) large pore (LP) and narrow pore (NP) configurations; (b) photographs of the pellets studied in this work; (c) the measured density of pellets as a function of applied nominal stress $\left(\sigma_{\text {nominal }}\right)$, labels on data points correspond to the applied weight reading on the press gauge.

Specular reflectance measurements are contingent on the surface quality of the sample. Therefore, the surfaces of each of the prepared pellets were characterized by electron microscopy 
and quantified using a non-contact optical profilometer and atomic force microscopy (see Fig. S4 and S5 in SI). Fig. S5k shows the three different measures of roughness (mean, rms and mean depth) determined using the $20 \times$ and the $50 \times$ optics on the profilometer to ensure that the optics were not affecting accuracy of the measurements. It is evident that the surface roughness of the pellets was low, showing less than $100 \mathrm{~nm}$ rms roughness across all the pellets. This is important for comparing the measured optical and dielectric properties: because the surface quality is unchanged across the pellets prepared under increasing applied stress, it is possible to conclude that the observed changes in the measured properties arise from evolution in the underlying framework structure of MIL-53(Al), as well as the increasing density, but independent of sample surface quality.

The Al-O octahedral sites in MIL-53(Al) have a strong affinity towards water, which results in LP to NP transformation when water molecules enter the pore; the crystal lattice undergoes a contraction from LP to NP. It is thus difficult to obtain purity of phase of MIL-53(Al) under ambient conditions - the moisture in the atmosphere is absorbed, resulting in a mixture of LP and NP configurations even if the material converted to pure LP (activated) phase through heating and evacuation prior to exposure to atmosphere. Indeed, because the pellets in this report were all prepared under ambient conditions, the precursor MIL-53(Al) powder contained an amount of its NP phase. We note that after the pellets were prepared they were only exposed to controlled laboratory air, so no increased amount of moisture was allowed to interact with the crystals.

The pellets were then studied using small-angle X-ray scattering (SAXS - see SI Fig. S3) and wide angle X-ray scattering (WAXS - see Fig. 2), to determine the crystal structure of the MIL53(Al) inside the pellets as a function of pelletizing pressure. We established that, with 
increasing pelletization stress the remaining crystalline material inside the prepared pellets approached purely NP phase plus the amorphized LP crystals. The LP and NP powder diffraction patterns were simulated using the CrystalMaker and CrystalDiffract software. ${ }^{29}$ Fig.2b shows the results of integration of the area underneath the largest LP and NP XRD peaks centered on $9^{\circ}$ (see inset marked \# in Fig.2a), which were used to track the changing amounts of the LP and NP phases with increasing stress. This analysis shows that the amount of LP phase decreases rapidly with increasing stress, whereas the NP content appears to remain constant. Further investigation shows that significant conversion from LP to NP phase does not occur (see SI section 9). We found that LP phase simply collapses under stress while NP phase withstands the level of stress applied in this study; we also found that this amorphous phase is not reversible. This finding is in line with theoretical studies showing improved mechanical properties of NP compared to LP. ${ }^{30}$ We suggest that LP crystals under stress are being converted to an amorphous LP phase. Literature on amorphous $\mathrm{MOFs}^{31}$ has shown that they lose long-range periodic order, but retain the basic building blocks and connectivity, ${ }^{32}$ including some porosity ${ }^{33}$ of their crystalline counterparts. As a result, the pellets studied using synchrotron IR radiation in this experiment appear to have the same amount of NP phase, but progressively less LP phase and more amorphous LP phase as the applied stress increases.
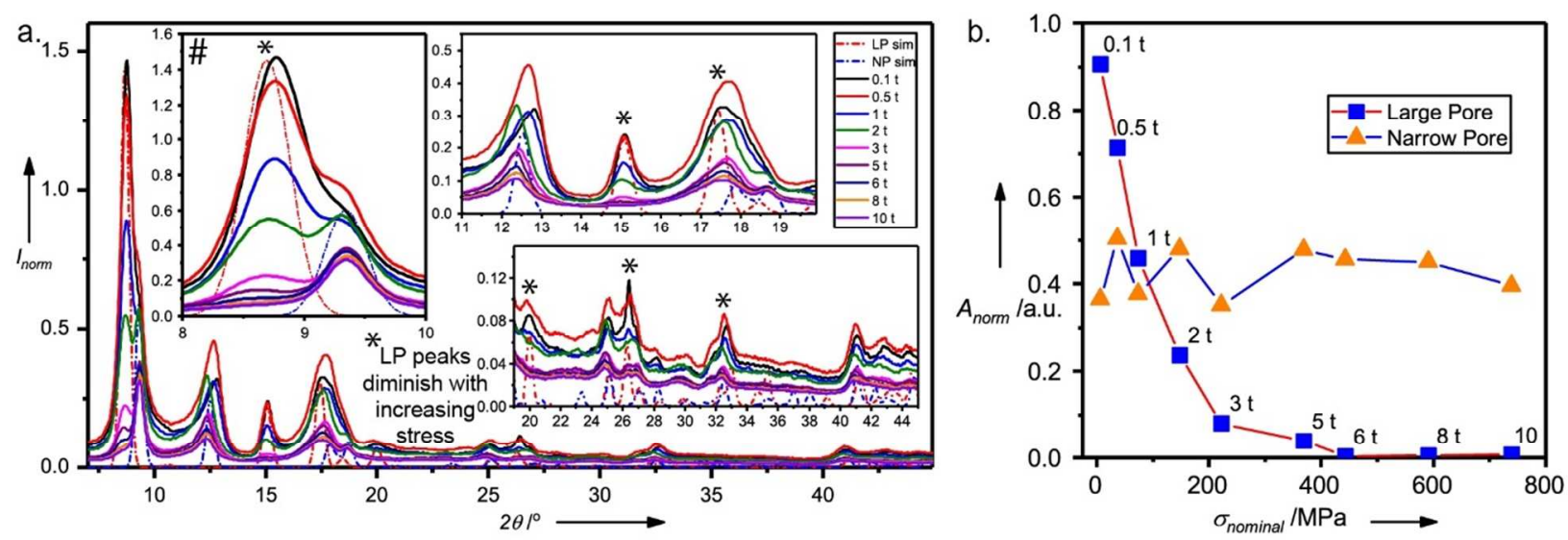

Figure 2. X-ray analysis of MIL-53(Al) pellets: (a) WAXS (XRD) patterns normalized to the 
product of density*thickness for all the pellets, with simulated powder XRD patterns for both the LP and NP crystal structures; the NP was simulated with a preferred orientation on the (200) plane with a factor of 0.524 in the CrystalDiffract software; (b) variation with applied stress of the areas of peaks fitted to the double peak marked \# in (a) associated with the LP and NP structures, respectively.

Drastic changes in the IR reflectance data, and the calculated dielectric properties, ${ }^{26}$ are observed as a result of the above described composition changes in the MIL-53(Al) pellets. Fig. S8 shows the collected reflectance $(R)$ spectra and SI section 10 discusses their necessary treatment as well as the applied Kramers-Kronig Transform (KKT). Fig.3 shows the complex dielectric functions of frequency: $\tilde{\varepsilon}(\omega)=\varepsilon^{\prime}(\omega)+i \varepsilon^{\prime \prime}(\omega)$, for each pellet, shown in the component form of spectra of its real $\left(\varepsilon^{\prime}\right)$ and imaginary $\left(\varepsilon^{\prime \prime}\right)$ parts (see Fig. S11 in SI for the complex refractive index). It can be seen that there is a stepwise decrease in $\varepsilon$ ' with increasing frequency of excitation. Specifically, each transition step exhibits a peak of varying magnitude associated with it, accompanied by distinct peaks in $\varepsilon '$, the latter describing dielectric losses. These steps are resonant vibrational responses of the material to the applied electromagnetic field. The orientational responses are detected at lower frequencies (THz phonons), ${ }^{5,7}$ while the electronic responses are observed at higher frequencies beyond $\sim 20 \mathrm{THz}$. It is important to note here that the spot size of the beam is of the order of $(100 \mu \mathrm{m})^{2}$, such that the measured reflectance spectrum is an average across this area, thus making the calculated properties the linear combination of properties of all the crystals and voids sampled in that area. 


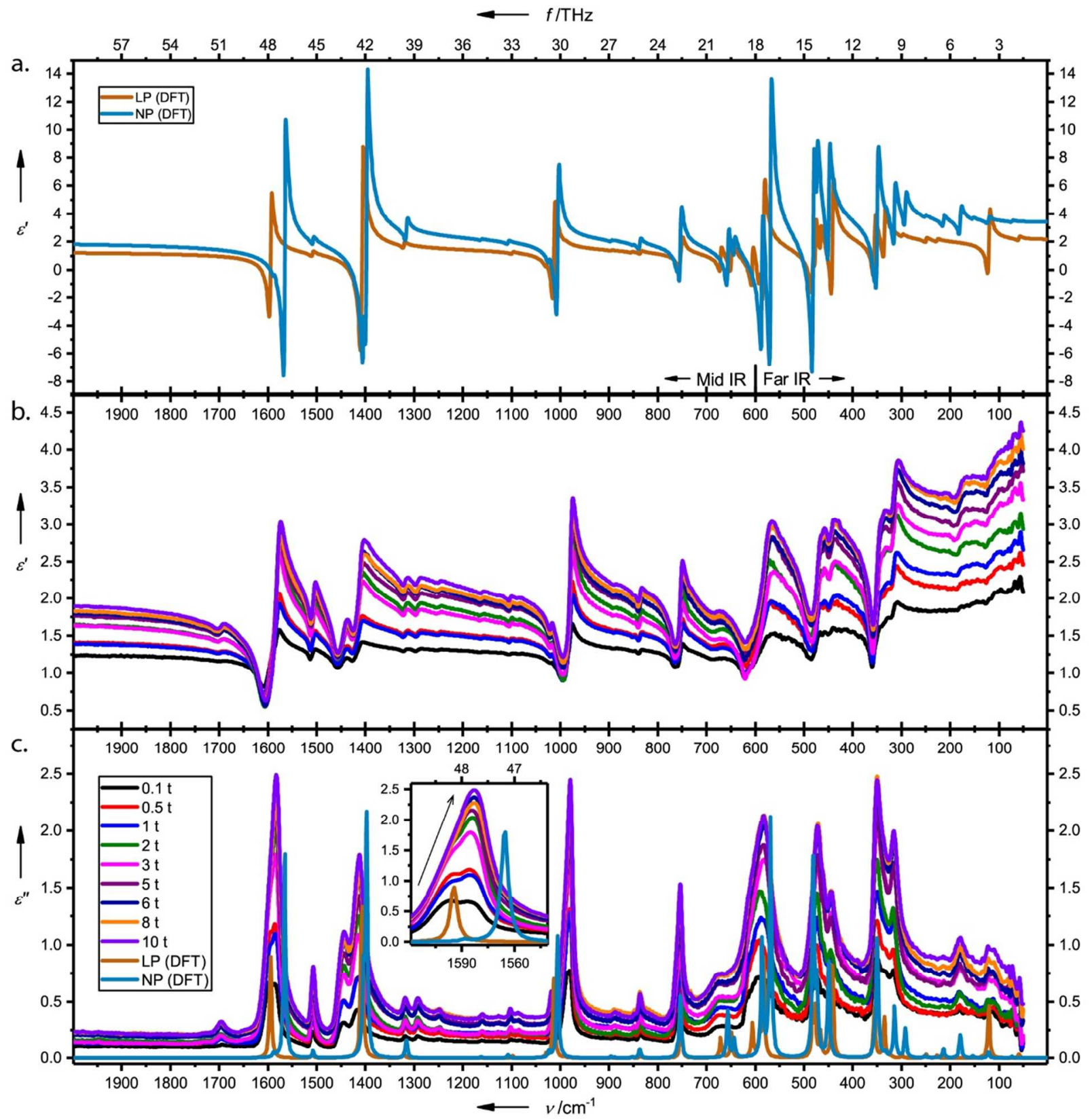

Figure 3. Complex dielectric functions of the MIL-53(Al) pellets: (a) real ( $\left.\varepsilon^{\prime}\right)$ and (c) imaginary $(\varepsilon ")$ parts of the DFT-calculated complex dielectric functions (see SI section 11 for DFT methods) of purely LP crystals and purely NP crystals supporting the experimentally obtained spectra. These are calculated from far- and mid-IR reflectance spectroscopy data (see Fig. S8 in SI for the collected reflectance spectra) via the Kramers-Kroenig relations, showing the (b) real part $\left(\varepsilon^{\prime}\right)$ and the (c) imaginary part ( $\left.\varepsilon^{\prime \prime}\right)$ of the complex dielectric function. See Fig. S9 and S10 in SI for detailed theory versus experiment comparison plots and for data up to $4000 \mathrm{~cm}^{-1}$. Note 
that $\varepsilon "$ of the DFT spectra here are scaled down to be comparable with the experimental data, so it is the positions and the relative intensities of the peaks that are important here, but not the absolute intensities.

Each of the loss $\varepsilon "$ " peaks grows with increasing pelletizing pressure and thus, as the above analysis of the pellet composition shows, with decreasing content of LP phase and increasing pellet density. These changes in amplitude are accompanied by changes in the shape of the peaks, which are indicative of changes occurring in the structure of the MIL-53(Al) crystals. Inset of Fig.3c shows one example of this transformation in the imaginary part of the dielectric function: the double peak around $1590 \mathrm{~cm}^{-1}(47.7 \mathrm{THz})$ grows dramatically with increasing applied stress as well as shifting the dominant peak area from $1600 \mathrm{~cm}^{-1}$ (48 $\left.\mathrm{THz}\right)$ down to $1585 \mathrm{~cm}^{-1}(47.6 \mathrm{THz})$. These changes are predicted by ab initio density functional theory (DFT) calculations of NP versus LP structures as can be seen in the same inset (for details of DFT calculations, see section 11 in SI). Detailed views of the other peaks of $\varepsilon$ " are presented in Fig.S10, which show similar shifts and intensity increases agreeing with DFT predictions of NP versus LP structures. Note that the DFT predicted $\varepsilon$ "' were scaled down so that they could be plotted together with the experimental spectra in an informative way while preserving the shape and relative intensities of the computed peaks.

The $\varepsilon "$ peaks grow rapidly from $0.1 \mathrm{t}$ to $5 \mathrm{t}$ applied load and level off, which is consistent with the decline in the LP fractional content in Fig. $2 \mathrm{~b}$ up to $5 \mathrm{t}$ followed by a complete loss of the LP phase. This spectroscopic evidence leads us to believe that the amorphous LP phase has a structure resembling the NP phase albeit without long-range periodicity (commonplace in amorphous $\mathrm{MOFs}^{31}$ ). Pellet density also increases rapidly over the same range before leveling off after $5 \mathrm{t}$ and approaching $90 \%$ of the theoretical NP crystal density. This finding suggests that the 
porosity of the amorphous LP phase is similar to the porosity of the NP phase. Meanwhile, the theoretical unit cell volume of a LP crystal is $1411.95 \AA^{3}$ and only $946.7 \AA^{3}$ in a NP crystal. Furthermore, the void space in those cells reduces from $54.4 \%$ of a LP unit cell to just $17.6 \%$ of a NP unit cell (these values are calculated using the Mercury CSD software). We thus claim that the porosity of the prepared pellets falls drastically with an increasing pelletization stress.

The real part of the dielectric function is tied to the imaginary part by Kramers-Kronig relations, so the factors affecting $\varepsilon^{\prime}$ are the same as those affecting $\varepsilon^{\prime \prime}$. Fig.3 shows the experimental $\varepsilon^{\prime}$ spectra as well as the $\varepsilon^{\prime}$ values predicted by DFT for LP and NP (see SI Fig. S9 for an overlapping $\varepsilon^{\prime}$ plot of DFT $v s$. experimental data). The predicted NP $\varepsilon^{\prime}$ is higher than that of LP and has higher amplitude resonances. The positions and relative intensities of the resonances captured by experiment agree well with the DFT predictions. Moreover, and most importantly, the rise in the values of $\varepsilon^{\prime}$ of the pellets with decreasing amounts of LP phase agrees with DFT predictions. We attribute this increase to the decrease in porosity of the MIL-53(Al) pellets from LP+NP to an (amorphized LP) $+\mathrm{NP}$ structure. Likewise, the transformation from LP to amorphous phase is essentially causing a fall in porosity of the crystals, thus further decreasing the porosity of the pellet. This is a neat outcome: a decrease in void volume significantly increases $\varepsilon^{\prime}$ of pellets with the same chemical composition of starting MIL-53(Al).

Above findings are of importance for the design of "low- $k$ " MOFs $(k$ here is interchangeable with $\varepsilon^{\prime}$ as used by various conventions, see discussion about the terminology in SI section 2$)^{26}$ with the design aim (among others) of keeping the real part of the complex dielectric function below $2 .{ }^{34}$ While the spectral range measured in the present experiments lies beyond the range of interest for current electronic technology (MHz-GHz), it is plausible to postulate that the observed effect of decreased porosity is similar beyond the range studied here, and possibly 
stronger at frequencies below $1 \mathrm{THz}$ since there appears to be a diverging trend being observed in the value of $\varepsilon$ ' with decreasing frequency of the applied electromagnetic field.

We thus show that larger pore size of a MOF material results in a lower real part of the complex dielectric function $\varepsilon^{\prime}$ (or $k$ as in other literature ${ }^{19,34}$ ). Therefore, when searching for low- $k$ dielectrics, it is prudent to explore those MOF variants, which maximize the pore size. Conversely, for high- $k$ dielectrics, the MOF variants that minimize pore size are likely to yield the best performance. In the light of this, a MOF structure that could reversibly and controllably switch between the LP $₹$ NP configurations will open the door to new generation of tunable dielectrics. In our opinion, MIL-53(Al) is an unlikely candidate for practical deployment in conventional electronics because it has a strong affinity for moisture uptake ${ }^{4}$ and due to synthetic challenges. ${ }^{35}$ Nevertheless, we established that in the region of 50-120 THz, the $\varepsilon$ ' of all prepared MIL-53(Al) pellets is strictly less than 2, and reaching as low as $k \sim 1.25$ for the pellet with the largest fraction of LP phase $(0.1 \mathrm{t})$. This result is remarkable and the reader is urged to be aware of the possible issue of inter-crystal voids, discussed in detail above, that might affect these figures. Furthermore, the bi-stability of MIL-53(Al) and the achievable high quality of pellets prepared from its powder are advantageous for further development of the method we demonstrated for studying dielectric properties of MOFs via specular reflection of synchrotron IR broadband radiation. This progress opens the door to future studies to accomplish "designer" MOF dielectrics and composite systems.

To conclude, we have demonstrated the efficacy of the specular reflectance method in conjunction with the use of a synchrotron light source for quantifying the detailed dielectric and optical properties of porous framework materials, the proposed approach will be applicable to polycrystalline powders, nanocrystals, nanosheets, etc. Fast acquisition of high-quality spectra is 
feasible ( minutes), making it possible to rapidly screen a large number of pelleted samples, which can accelerate the development of MOF dielectrics. We also demonstrate excellent agreement of experimental complex dielectric function data with theoretical DFT calculations, which paves the way towards advancing MOF designs, from the bottom-up in the important field of dielectrics.

EXPERIMENTAL METHODS: Activated MIL-53(Al) polycrystalline powder (Basolite A100) was purchased from Sigma-Aldrich and used as received. Pellets with an averaged thickness of $\sim 1 \mathrm{~mm}$ were prepared on a standard hydrostatic lab press with a die diameter of $13 \mathrm{~mm}$. All pellets were then characterized via X-ray scattering in transmission mode using the Xenocs NanoInXider (R53 Materials Characterization Laboratory, ISIS) equipped with two 'Pilatus 3' 2-D detectors for SAXS and WAXS. All X-ray spectra were collected for $300 \mathrm{~s}$ under high-resolution beam settings: $400 \mu \mathrm{m}$ spot size on sample and $15 \mathrm{Mph} / \mathrm{s}$ typical flux. The physical density of each pellet was determined by weighing each pellet and dividing this quantity by its nominal volume (dimensions via a micrometer). The collected X-ray scattering intensities were normalized by density*thickness of the corresponding pellets. Details about the synchrotron beamline settings for the IR specular reflectance measurements are given in SI section 1. Details of DFT calculations are given in SI section 11.

\begin{abstract}
ACKNOWLEDGEMENTS: We acknowledge the Diamond Light Source for the provision of beamtime SM14902 at B22 MIRIAM. K.T. thanks the Balliol College Scholarship for supporting his postgraduate studies. J.C.T. thanks the Engineering and Physical Sciences Research Council (EPSRC) for research funding (EP/N014960/1). M.R.R. acknowledges the
\end{abstract}


EPSRC DTA and STFC CMSD Award (13-05) for postgraduate funding. A.K.C. thanks the Samsung GRO for postgraduate funding. We thank the Research Complex at Harwell (RCaH), Oxfordshire, for access to advanced materials characterization suite. We are grateful to Dr. Gavin Stenning and Dr. Marek Jura (R53 Materials Characterization Lab) at the ISIS Rutherford Appleton Laboratory, for the X-ray characterization facilities.

Supporting Information Available: a detailed description of the measurements procedures at B22 of Diamond Light Source; a discussion of notation conventions in the field of dielectrics; data on the surface quality of the prepared pellets; a discussion on the physical phenomena at play in specular reflection off a MOF pellet; the measured reflectance spectra; detailed comparisons of measured $\varepsilon$ ' against values simulated via Density Functional Theory (DFT); detailed comparisons of measured $\varepsilon "$ against values simulated via DFT; plot of the complex refractive indices for all pellets; details of a further pelletisation study for the Sigma supplied MIL-53(Al) crystals; details of the Kramers-Kronig transform used in this stud and its MATLAB implementation; and details of the DFT calculations.

\section{REFERENCES}

1. Furukawa, H.; Cordova, K. E.; O'Keeffe, M.; Yaghi, O. M. The chemistry and applications of metal-organic frameworks. Science 2013, 341, 1230444.

2. Senkovska, I.; Kaskel, S. Ultrahigh porosity in mesoporous MOFs: promises and limitations. Chem. Commun. 2014, 50, 7089-7098.

3. Wharmby, M. T.; Henke, S.; Bennett, T. D.; Bajpe, S. R.; Schwedler, I.; Thompson, S. P.; Gozzo, F.; Simoncic, P.; Mellot-Draznieks, C.; Tao, H., et al. Extreme Flexibility in a Zeolitic Imidazolate Framework: Porous to Dense Phase Transition in Desolvated ZIF-4. Angew. Chem. Int. Ed. 2015, 54, 6447-6451.

4. Ferey, G.; Serre, C. Large breathing effects in three-dimensional porous hybrid matter: facts, analyses, rules and consequences. Chem. Soc. Rev. 2009, 38, 1380-1399. 
5. Ryder, M. R.; Civalleri, B.; Bennett, T. D.; Henke, S.; Rudic, S.; Cinque, G.; FernandezAlonso, F.; Tan, J. C. Identifying the role of terahertz vibrations in metal-organic frameworks: from gate-opening phenomenon to shear-driven structural destabilization. Phys. Rev. Lett. 2014, $113,215502$.

6. Comotti, A.; Bracco, S.; Sozzani, P. Molecular Rotors Built in Porous Materials. Acc. Chem. Res. 2016, 49, 1701-1710.

7. Ryder, M. R.; Civalleri, B.; Cinque, G.; Tan, J. C. Discovering connections between terahertz vibrations and elasticity underpinning the collective dynamics of the HKUST-1 metal-organic framework. CrystEngComm 2016, 18, 4303-4312.

8. Horike, S.; Shimomura, S.; Kitagawa, S. Soft porous crystals. Nat. Chem. 2009, 1, 695-704.

9. Lew, C. M.; Cai, R.; Yan, Y. Zeolite thin films: from computer chips to space stations. Acc. Chem. Res. 2010, 43, 210-219.

10. Slater, A. G.; Cooper, A. I. Porous materials. Function-led design of new porous materials. Science 2015, 348, aaa8075.

11. Dinca, M.; Leonard, F. Metal-organic frameworks for electronics and photonics. MRS Bull. 2016, 41, 854-857.

12. Stavila, V.; Talin, A. A.; Allendorf, M. D. MOF-based electronic and opto-electronic devices. Chem. Soc. Rev. 2014, 43, 5994-6010.

13. Chaudhari, A. K.; Ryder, M. R.; Tan, J. C. Photonic hybrid crystals constructed from in situ host-guest nanoconfinement of a light-emitting complex in metal-organic framework pores.

Nanoscale 2016, 8, 6851-6859.

14. Dolgopolova, E. A.; Shustova, N. B. Metal-organic framework photophysics: Optoelectronic devices, photoswitches, sensors, and photocatalysts. MRS Bull. 2016, 41, 890-895.

15. Walsh, A.; Butler, K. T.; Hendon, C. H. Chemical principles for electroactive metal-organic frameworks. MRS Bull. 2016, 41, 870-876.

16. Ryder, M. R.; Tan, J. C. Nanoporous metal organic framework materials for smart applications. Mater. Sci. Tech. 2014, 30, 1598-1612.

17. Leong, C. F.; Usov, P. M.; D'Alessandro, D. M. Intrinsically conducting metal-organic frameworks. MRS Bull. 2016, 41, 858-864.

18. Talin, A. A.; Centrone, A.; Ford, A. C.; Foster, M. E.; Stavila, V.; Haney, P.; Kinney, R. A.; Szalai, V.; El Gabaly, F.; Yoon, H. P., et al. Tunable electrical conductivity in metal-organic framework thin-film devices. Science 2014, 343, 66-69.

19. Usman, M.; Mendiratta, S.; Lu, K. L. Metal-Organic Frameworks: New Interlayer Dielectric Materials. ChemElectroChem 2015, 2, 786-788. 
20. Zagorodniy, K.; Seifert, G.; Hermann, H. Metal-organic frameworks as promising candidates for future ultralow-k dielectrics. Appl. Phys. Lett. 2010, 97, 251905.

21. ITRS Roadmap (www.itrs2.net).

22. Eslava, S.; Zhang, L. P.; Esconjauregui, S.; Yang, J. W.; Vanstreels, K.; Baklanov, M. R.; Saiz, E. Metal-Organic Framework ZIF-8 Films As Low-kappa Dielectrics in Microelectronics. Chem. Mater. 2013, 25, 27-33.

23. Usman, M.; Lee, C. H.; Hung, D. S.; Lee, S. F.; Wang, C. C.; Luo, T. T.; Zhao, L.; Wueg, M. K.; Lu, K. L. Intrinsic low dielectric behaviour of a highly thermally stable Sr-based metalorganic framework for interlayer dielectric materials. J. Mater. Chem. C 2014, 2, 3762-3768.

24. Redel, E.; Wang, Z. B.; Walheim, S.; Liu, J. X.; Gliemann, H.; Woll, C. On the dielectric and optical properties of surface-anchored metal-organic frameworks: A study on epitaxially grown thin films. Appl. Phys. Lett. 2013, 103, 091903.

25. Warmbier, R.; Quandt, A.; Seifert, G. Dielectric Properties of Selected Metal-Organic Frameworks. J. Phys. Chem. C 2014, 118, 11799-11805.

26. See the Supporting Information (SI) for details of methods and details of data analysis.

27. Walker, A. M.; Civalleri, B.; Slater, B.; Mellot-Draznieks, C.; Cora, F.; Zicovich-Wilson, C. M.; Roman-Perez, G.; Soler, J. M.; Gale, J. D. Flexibility in a metal-organic framework material controlled by weak dispersion forces: the bistability of MIL-53(Al). Angew. Chem. Int. Ed. 2010, 49, 7501-7503.

28. Yot, P. G.; Yang, K.; Guillerm, V.; Ragon, F.; Dmitriev, V.; Parisiades, P.; Elkaim, E.;

Devic, T.; Horcajada, P.; Serre, C., et al. Impact of the Metal Centre and Functionalization on the Mechanical Behaviour of MIL-53 Metal-Organic Frameworks. Eur. J. Inorg. Chem. 2016, 44244429.

29. CrystalMaker Software ver.9 (www.crystalmaker.com).

30. Wang, M.; Zhang, X.; Chen, Y.; Li, D. How Guest Molecules Stabilize the Narrow Pore Phase of Soft Porous Crystals: Structural and Mechanical Properties of MIL-53(Al) $\supset$ H2O. J. Phys. Chem. C 2016, 120, 5059-5066.

31. Bennett, T. D.; Cheetham, A. K. Amorphous metal-organic frameworks. Acc. Chem. Res. 2014, 47, 1555-1562.

32. Bennett, T. D.; Goodwin, A. L.; Dove, M. T.; Keen, D. A.; Tucker, M. G.; Barney, E. R.; Soper, A. K.; Bithell, E. G.; Tan, J. C.; Cheetham, A. K. Structure and properties of an amorphous metal-organic framework. Phys. Rev. Lett. 2010, 104, 115503.

33. Chapman, K. W.; Halder, G. J.; Chupas, P. J. Pressure-induced amorphization and porosity modification in a metal-organic framework. J. Am. Chem. Soc. 2009, 131, 17546-17547. 
34. Stassen, I.; Burtch, N.; Talin, A.; Falcaro, P.; Allendorf, M.; Ameloot, R. An updated roadmap for the integration of metal-organic frameworks with electronic devices and chemical sensors. Chem. Soc. Rev. 2017, 46, 3185-3241.

35. Mounfield, W. P., 3rd; Walton, K. S. Effect of synthesis solvent on the breathing behavior of MIL-53(A1). J. Colloid Interface Sci. 2015, 447, 33-39. 